\title{
Um Framework para Extracão de Dados em Documentos Científicos: Uma Abordagem baseada em XML
}

\section{Davi Medeiros Cabral, Roberto Souto Maior de Barros}

Centro de Informática - Universidade Federal de Pernambuco (UFPE)

Caixa Postal 7.851 - 50.732-970 - Recife - PE - Brazil

$$
\text { \{dmc3, roberto\}@cin.ufpe.br }
$$

Abstract. Nowadays, it is very easy to publish documents in the Internet. Thus, a large number of documents become available every day, making the management and manipulation of these documents progressively more difficult. Moreover, the existence of several incompatible formats makes the development of efficient tools to deal with such documents a very complex task. This paper proposes a semantic restructure framework for the conversion of documents to XML, to make it possible the consistent use of query and transformation languages.

Resumo. Hoje em dia, documentos são facilmente publicados através da Internet. Assim, um grande número de documentos torna-se avaliados diariamente, fazendo o gerenciamento e a manipulação desses progressivamente mais difícil. Além disso, a existência de formatos incompatíveis faz o desenvolvimento de ferramentas eficientes para o tratamento com documentos uma tarefa altamente complexa. Este artigo propõe um framework de reestruturação semântica para a conversão de documentos para XML, fazendo possivel o uso consistente de linguagens de consulta e transformação.

\section{Introdução}

Com a explosão da World Wide Web, uma grande quantidade de dados em diferentes assuntos e formatos tem se tornado disponível on-line. Porém, esses dados geralmente encontram-se de uma maneira semi-estruturada ou não-estruturada, tornando as consultas dos usuários limitadas a técnicas de browsering ou busca por palavras-chaves. Essas técnicas não são tão eficientes, pois podem retornar uma grande quantidade de documentos irrelevantes (precision) ou ainda documentos relevantes podem não ser retornados (recall) [Aldea 2003]. Além disso, a tarefa de extração das informações fica a cargo da atividade humana, o que inviabiliza a Web Semântica de [Berners-Lee 2001].

Muitos estudos tentam dar à manipulação em documentos o mesmo poder dos SGBDs, dentre as quais pode-se citar: (1) a criação de uma linguagem de consulta para documentos Web (ex. [Arocena 1998]) e (2) a popularização de um banco de dados a partir de dados extraídos de fontes da Internet, possibilitando tratamento posterior.

Uma solução comum para essa última estratégia é o desenvolvimento de programas especializados, denominados wrappers, criados para identificar dados de fontes da Web e mapeá-los para um formato apropriado. 
Os wrappers fornecem o suporte necessário para alcançar consultas precisas e eficientes em documentos. Porém, a maioria deles sofre por confiar na ord dos dados ou na estrutura dos documentos (ex. HTML) da Internet, acarretando grande esforço de manutenção quando características estruturais são modifica ([Embley 1999]). Além disso, as abordagens estudadas realizam extração de da apenas em documentos nos formatos HTML, XML ([Bray 2004]) ou texto, esquecend se que a Internet é muito mais heterogênea, abrangendo formatos como PDF, DO LaTeX, etc. Um outro ponto diz respeito aos núcleos dos wrappers que em um mesmo sistema normalmente são semelhantes para diferentes fontes de informaça ocasionando duplicações de código e, conseqüentemente, de esforço de programação.

Este artigo busca solucionar os problemas citados propondo um framework para extração e reestruturação de dados que otimize reuso e extensão para diferentes formatos de documentos científicos na Web. A flexibilidade da ferramenta é alcançad a partir da modularização e da comunicação de componentes através de XML.

O restante deste documento está organizado da seguinte forma: a seção dois apresenta uma breve discussão sobre técnicas e modelos de sistemas para extração de dados; a seção três apresenta o modelo proposto e sua implementação. Por último, seção quatro fala sobre as conclusões e trabalhos futuros.

\section{Trabalhos Relacionados}

O desenvolvimento manual de wrappers possui barreiras bem conhecidas. Atualmente, muitas propostas têm surgido para solucionar essas dificuldades, sendo classificadas aqui de acordo com o tipo de técnica de extração e com sua utilização.

\subsection{Mecanismos de Extração}

Segundo [Laender 2002], uma ferramenta de geração de wrapper pode ser classificada de acordo com seis diferentes técnicas de extração utilizadas. Porém, por questão de espaço e escopo de trabalho apenas as técnicas extração NLP, Indução e Ontologia voltadas para documentos livres e semi-estruturados serão apresentadas.

Ferramentas baseadas em Processamento de Linguagem Natural (NLP) buscam obter regras de extração de dados em documentos escritos em linguagem natural, aplicando técnicas de filtragem, tipos de palavras e classes semânticas. Essas técnicas são utilizadas na construção de relacionamentos entre frases e sentenças de elementos utilizados na derivação de regras de extração. Como exemplos de ferramentas podem ser citados o SRV [Freitag 2000] e o WHISK [Soderlan 1999]

Ferramentas de indução de Wrapper geram regras de extração baseadas em delimitadores (prefixos e sufixos) derivados de um dado conjunto de exemplos de treinamento. Diferentemente das ferramentas NLP, essa abordagem confia em características de formatação que implicitamente descrevem a estrutura de pedaços de dados encontrados - ferramentas representativas são o WIEN [Kushmerick 2000] e 0 STALKER [Muslea 2001].

Ferramentas baseadas em ontologia utilizam uma ontologia de domínio específico para localizar constantes presentes em um documento e assim construil objetos com elas. Uma ontologia é "uma especificação explícita e formal de conceitos" ([Gruber 1993]), um meio de representação do conhecimento. Essa abordagem difer mais, por não confiar na estrutura de apresentação dos dados nos doctrmentos. das demais, por nais flexível e abrangente que as demais abordagens, porém possui uma Assim, ela e mais flexas menos automatizada. Um exemplo dessa abordagem é o sistema aprendizagem de rembley 1999].

apresentado em [Embley 1999]

\section{Utilização de Wrappers}

exáo traz uma visão geral da utilização de wrappers em sistemas de extração de Essa squi serão definidas três estruturas evolutivas de utilização de dados em docu ser desenvolvidas utilizando, por exemplo, wrappers (vê tigura 1 , and-alone.

agentes ou aplicações sland-alone.

A abordagem simples (figura la) é a mais elementar, pros de um documento a conversão direta entre dois formatos difecis HTML para tuplas de um SGr

abordagem é [Embley 1999].

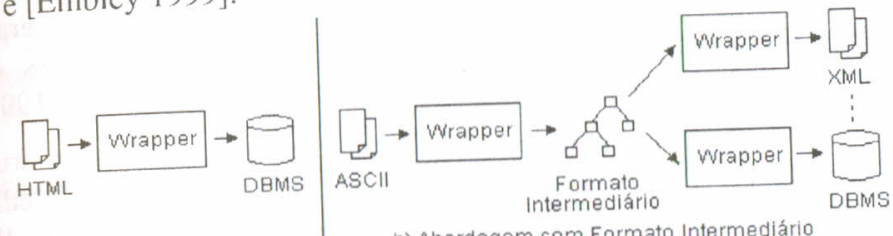

a) Abordagem Simples momato Intermediário DMS

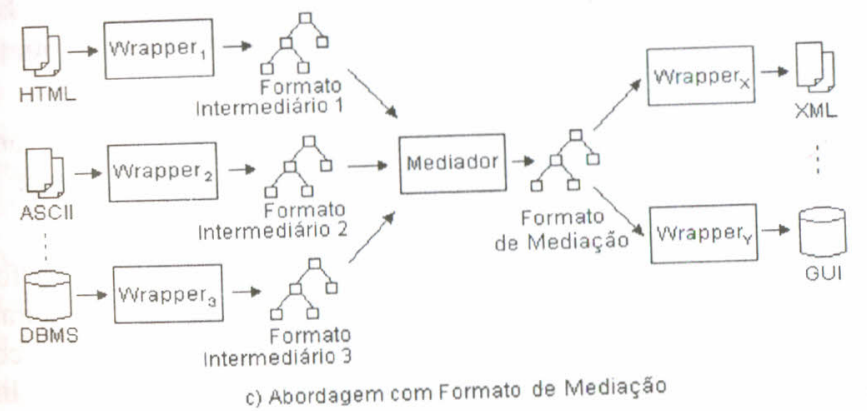

Figura 1 - Abordagens de utilização de wrappers

Para tornar a estrutura anterior mais flexível, é adicionado a ela um formato de representação intermediário para os dados extraídos (figura 1b). Esse formato pode ser uma interface de um arquivo (ex. XML, OEM [Hammer 1997]) uma interface de manipulação de objetos, um arquivo (ex. XML, OEM [Hammer 1997]) etc. Essa modificação possibilita estender facilmente o sistema de extração com formatos diferentes de saídas de dados, a partir de wrappers simples, que convertem formato intermediário interno em um outro.

A estratégia da figura 1c amplia a abordagem anterior para diferentes tipos de entradas. Nesse caso, surge um componente especializado, denominado mediador, responsável por caso, surge um componente entre os formatos gerados pelos wrapper. de entrada e a interface de entrada padrão para os wrappers de saída. Portanto, para adiciona ser alterado ou empilhado adicionar outro wrapper de entrada, o módulo mediador deve ser alterado ou empithácio. a um outro, desenvolvido para o reconhecimento desse novo formato inter 
Ainda com relação a essa estratégia, pode-se notar que os formato intermediários possuem em comum a forma de representação dos dados extraídos (e XML, OEM), mas diferem na estrutura de como esses dados são modelados (ex granularidade, relacionamento entre dados etc.). Essa peculiaridade ocasiona aumento de esforço significativo no desenvolvimento dos mediadores, obrigando-os solucionar heterogeneidades estruturais e semânticas dos dados ([Wache 2001]). Um exemplo de ferramenta de extração desse tipo é o TSIMMIS [Hammer 1997].

\section{Modelo Proposto}

Essa seção apresenta o framework proposto e para tanto é preciso entender o que es termo significa. Um framework tem por objetivo auxiliar a construção de aplicações complexas pertencentes a um determinado domínio, através da definição de um modelo que possibilite a reutilização de código. Um dos pontos essenciais de um framework é definição de suas funcionalidades frozen spots e hot spots. As frozen spots são as funcionalidades fixas, comuns ao domínio, constituindo a parte inalterada de um framework. Por outro lado, as funcionalidades hot spots são aqueles em aberto específicas de cada domínio, e que necessitam ser implementadas [Fayad 1999]

O framework proposto (vê figura 2) é baseado na abordagem de estruturação de wrappers da figura 1c, sendo diferenciada pela ausência do módulo Mediador, pela adição do módulo Alimentador de Conhecimento e pela quebra do módulo Wrapper de Entrada em três outros: Wrapper Simples de Entrada, Reestruturador e Extrator de Dados. Essa estratégia modular torna mais flexível o problema de extração, permitindo assim a reutilização do módulo de extração de dados.

$\mathrm{Na}$ abordagem da figura 2, os Wrappers de Entradas são mecanismos de encapsulamento e reuso de ferramentas existentes para conversão entre diferentes formatos.

Os módulos Reestruturadores são utilizados na padronização do formato de apresentação dos documentos, solucionando heterogeneidades estruturais. Esses módulos diferem-se do módulo mediador da figura 1c por não gerenciar consultas a dados em diferentes fontes (Wrappers de Entrada). Um Reestruturador limita-se a converter o que é retornado por um Wrappers de Entrada em arquivos (XML Unificado) usando conceitos semanticamente pobres como parágrafo, figura, tabela etc., tornando essa estratégia menos complexa do que as de desenvolver ou modificar um mediador

O módulo Extrator de Dados implementa uma das técnicas apresentadas na seção 2.1, sendo o responsável pela identificação e obtenção de informações semanticamente ricas como autor, orientador, título, ano de publicação e referência bibliográfica, entre outras. Os metadados necessários a esse processo da extração são retirados da base externa de conhecimento, escrita em XML. Esse é o módulo mais complexo do modelo e, por isso, a preocupação em projetá-lo de maneira centralizada.

Por último, o Alimentador de Conhecimento é o responsável pela alimentação semi-automática da base de conhecimento do sistema e os wrappers de saída são 0 mesmos apresentados na figura $1 \mathrm{c}$

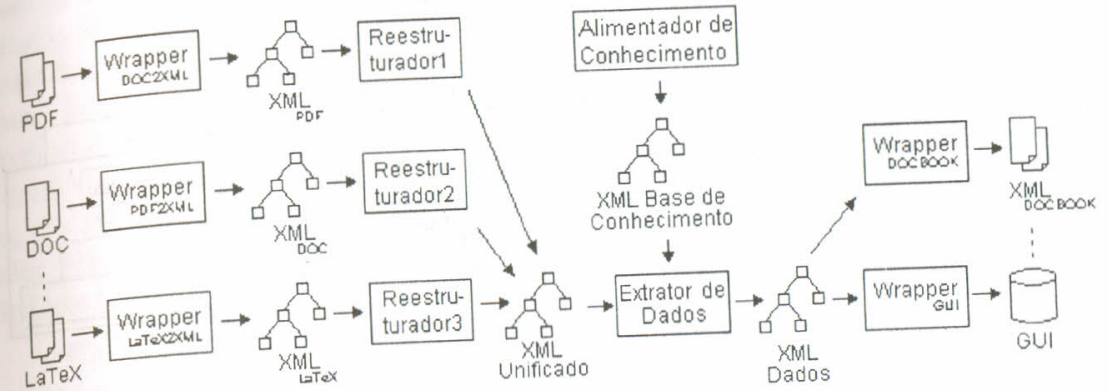

Figura 2 - Modelo de sistema de extração para documentos científicos

or serem gerais e estáveis, os módulos Extrator de Dados e Alimentador de Prozen spots, enquanto que os demais módulos são Conhecimento constionalidades hot spots do framework.

responsáveis pelas funcionalidades

implementação foi utilizada como módulo de conversão de documentos científicos A inp necessário ao sistema de gerenciamento e publicação de teses e [Santos 2002]. A escolha por esse tipo de documento for dissertações proposto por [Santos 2002]. A escolha por esse tipo de doc acarreta na devido a sua riqueza de informações e formato não-estruturado, que a maioria dos dispersão das informações dentro do texto. Essa caracteristica é presentenvido na documentos Web, possibilitando a utilização do conhecimento documentos com outros tipos de conto.

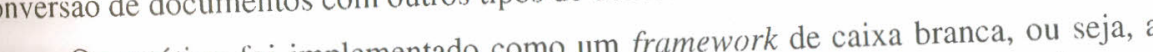
O protótipo foi implementado como um framework de caixa bran para cada novo partir da definição de classes abstratas que deven ser especia Template Method.

caso. Em geral, essas classes são definidas utilizando o padrão Templá do módulo Alimentador de

Para cada módulo do modelo proposto, com exceção do módulo Alimentador de Conhecimento, foi implementado um protótipo. Os módulos foram desenvolvidos tilizando a linguagem Java, dando origem aos seguintes pacotes (figura 3) utilizando a linguagem Java, dando WrapperEntrada, Reestruturador, MaquinaExtracao e Wrappersaida. A manipung (escrita e leitura) dos documentos XMLs entre os módulos foi realizada utilizando data binding Castor [Gilmartin 2002], cujos modelos de objetos para a representação dos bubacotes ModeloUnificado, dos dados e os respectivos parsers foram definidos nóltimos ModeloDados, ModeloEntrada e ModeloSaida. Adicionalmente, esses dor utilizado subpacotes devem ser expandidos para cada novo formato de arquivo a ser utilizado pelo sistema. 


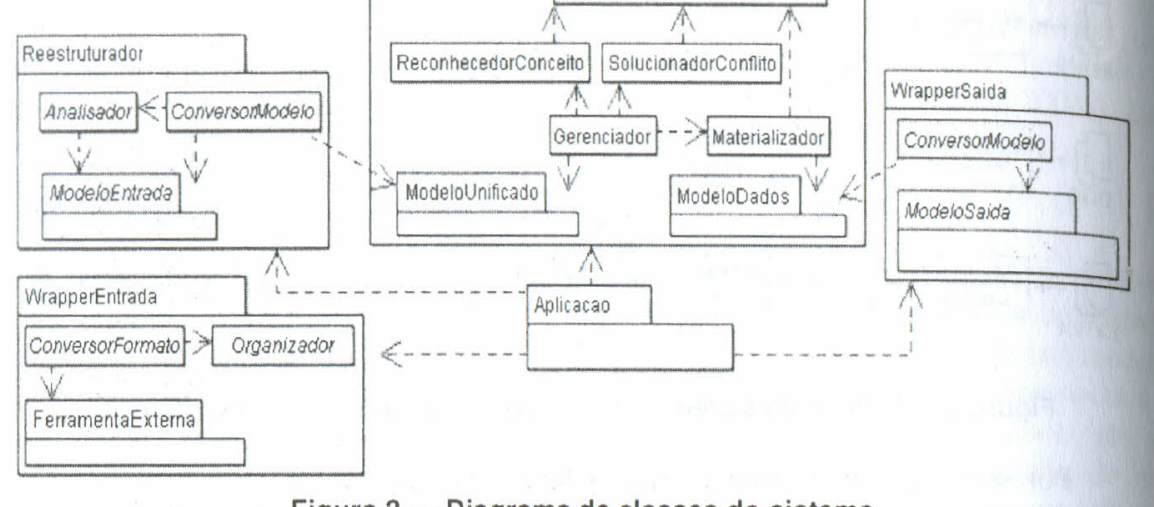

Figura 3-Diagrama de classes do sistema

O módulo WrapperEntrada é composto basicamente pelas classes abstratas ConversorFormato e Organizador, que deverão ser estendidas para cada novo formato de entrada suportado. A classe ConversorFormato é a responsável por encapsular uma ferramenta de conversão, pacote FerramentaExterna, que transforme arquivos em formato específico para XML. Baseada na idéia do HTML Tidy [Raggett 1994] para correção de arquivos HTML, a classe Organizador deve ser implementada para a correção dos possíveis XMLs mal formados - gerados pelo ConversorFormato. Os formatos de arquivos de entrada implementados para o sistema foram DOC e PDF através do encapsulamento das ferramentas de conversão Majix ${ }^{2}$ e Pdftohtml ${ }^{3}$, respectivamente.

Dando continuidade aos módulos, o pacote Reestruturador é dividido nas classes abstratas Analisador e ConversorModelo. A primeira classe é responsável por analisar o arquivo XML, gerado por um wrapper de entrada, e por quantificar a ocorrência de certos atributos como distância entre linhas e valores para a margem esquerda, por exemplo. O ConversorModelo utiliza os valores retornados pelo Analisador e aplica regras heurísticas para reestruturar o documento em um XML Unificado definido na seção 3. Isso foi necessário porque, por exemplo, a ferramenta Pdftohtml retornava um documento XML sem o conceito de parágrafos, dividindo uma possível informação em linhas diferentes.

Com relação ao pacote MaquinaExtracao, a estratégia utilizada foi baseada no uso de ontologia proposto em [Embley 1999]. O módulo MaquinaExtracao é composto pelas classes ReconhecedorConceito, SolucionadorConflito, Gerenciador e Materializador, além dos subpacotes ModeloConhecimento, ModeloUnificado e ModeloDados

A abordagem de extração de dados consiste de cinco etapas: (1) criação de um modelo ontológico sobre uma área de interesse, subpacote ConversorModelo; (2) btenção do texto não-estruturado, mas com parágrafos, através de uma solicitação ao método getText ; (3) invocação da classe Reconhecedor Conceito que usa recur de combinação informadas na análise heurística as reg de cada documento não-estruturado os objetos

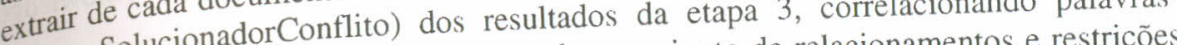
(classe Solucionadortantes extraídas, usando o conjunto de relacionamentos e restriçoes chaves com as constantes exia - para se determinar quais dados serão considerados ou de cardinalidade da ontologia do SolucionadorConflito para composição do XML de ão: (5) análise dos resultados do SolucionadorContitó para compos pão do Xuestrados não, (5) anelo Materializador. Adicionalmente, os quatro últimos passos são orquestrado pelo Gerenciador. XML para um formato de saída específico (subpacote ModeloSaida) como, Essa operação é realizada por uma classe por exemplo, registros em um banco de dados. Essa o do pacote Reestruturador, definida abstrata, nomeadicação do padrão Builder.

\section{Conclusões e Trabalhos Futuros}

(a) wrappers, culminando na apresentação e Nesse artigo, foi realizado um estudo sobre wrappers, implementação de um novo modelo para sistema dá o suporte necessário ao desenvorvine proposto o renciamento, consulta e publicação de documentos. Aler o gerenciam classificação independentes (DocBook[Walsh

2003]), bem como modelos nacionais e internacionais (ex. ABNT).

O desenvolvimento desse sistema mostrou a flexibilidade trazida pelo uso de XML como linguagem de representação de metadados e de comunicação entre os módulos do protótipo. Além disso, a representação em XML dos dados extraídos, jức juntamente considerável variedade de documentos em rápida de wrappers de saída pa

desenvolvido encontra-se implementado apenas para os formatos DOC e PDF. Futuramente pretende-se realizar a expansão do sistema para (ex. LaTeX, XHTML etc.) e publicações (ex. livros, atroc feitas melhorias como, por exemplo, a utilização artigos, etc.). Além disso, poderiam ser feitas mecthorias como, por exemplo, a utilização de parser DOM Level 3 na manipulação de arquivos XML e de Web services no desenvolvimento dos módulos do sistema, contribuindo respectivamente na eliminação dos problemas

Com relação à base de conhecimento, poderia ser realizado um estudo sobre a viabilidade de representação do seu conhecimento em XMI, o que permitiria a alimentação da mesma a partir de uma ferramenta de modelagem UML (ex. ArgoUML) ou de um módulo alimentador de conhecimento utilizando técnicas de aprendizagem da Inteligência possível a utilização de outras técnicas de extração de dados como, por exemplo, NLP ou indução. 


\section{References}

Aldea, A., Bañares-Alcántara, R., Bocio, J., Gramajo, J., Isern, D., Kokossis, A Jiménez, L., Moreno, A. and Riaño, D. (2003) "An Ontology-Based Knowled, Management Platform”, In: $18^{\text {th }}$ IJCAI. IIWeb-03.

Arocena, G. and Mendelzon, A. (1998) "WebOQL: Restructing Documents, Databases, and Webs", In: Proceedings of the $14^{\text {th }}$ ICDE. Orlando, Florida.

Berners-Lee, T., Hendler, J. and Lassila, O. (2001) "The Semantic Web", In: Scientific American.COM., http://www.sciam.com/print_version.cfm?articleID $=00048144$ 10D2-1C70-84A9809EC588EF21.

Bray, T., Paoli, J., Sperberg-McQueen, C., Maler, E. and Yergeau, F. (2004) "Extensible Markup Language (XML) 1.0 (Third Edition)", In: W3C Recommendation, http://www.w3.org/TR/2004/REC-xml-20040204.

Embley, D., Campbell, D., Jiang, Y., Liddle, S., Lonsdale, D., Ng, Y. and Smith, R. (1999) "Conceptual-Model-Based Data Extraction from Multiple-Record Web Pages".

Fayad, M., Schmidt, D., Johnson, R. (1999) "Building Application Frameworks: Object-Oriented foundations of frameworks design". Wiley, Estados Unidos.

Freitag, D. (2000) "Machine Learning for Information Extraction in Informal Domains", In: Machine Learning 39.

Gilmartin, A., Horowitz, R. and Suez, E. (2002) "Castor: An Open Source Data Binding Framework for Java", In: ExoLab Group, http://www.castor.org, February.

Gruber, T. (1993) "A translation approach to portable ontology specifications", In: Kknowledge Acqui-sition, 5(2):199-220

Hammer, J., McHugh, J. and Gracia-Molina H. (1997) "Semistructured Data: The TSIMMIS Experience", In: Proceedings of the $1^{\text {st }}$ ADBIS. St. Petersburg, Russia.

Kushmerick, N. (2000) "Wrapper Induction: Efficiency and Expressiveness", In: Artificial Intelligence Journal 118.

Laender, A., Ribeiro-Neto, B., Silva, A. and Teixeira, J. (2002) "A Brief Survey of Web Data Extraction Tools".

Muslea, I., Minton, S. and Knoblock, C. (2001) "Hierarchical wrapper induction for semistructured information sources", In: Autonomous Agents and Multi-Agent 4.

Raggett, D. (1994) "Clean up your Web pages with HTML TIDY", http://www.w3.org/People/Raggett/tidy, January.

Santos, H., Batista, M. and Barros, R. (2002) "Publishing Theses and Dissertations: An Approach Using XML", In: The $4^{\text {th }}$ IIWAS.

Soderlan, S. (1999) "Learning Information Extraction Rules for Semi-Structured and Free Text", In: Machine Learning 34.

Wache, H., Vögele, T., Visser, U., Stuckenschmidt, H., Schuster, G., Neumann, H. and Hübner, S. (2001) "Ontology-Based Integration of Information - A Survey of Existing Approaches".

Walsh, N. and Muellner, L. (2003) "DocBook: The Definitive Guide". O'Reilly. 